\title{
Sex Differences in Overlapping Chronic Non-cancer Pain Conditions in a Tertiary Pain Clinic
}

Samah Hassan ${ }^{1,3 *}$, Allan Gordon ${ }^{2}$ and Gillian Einstein ${ }^{1,3}$

${ }^{1}$ Institute of Medical Sciences, University of Toronto, Toronto, ON M5S, Canada

${ }^{2}$ Wasser Pain Management Centre, Mount Sinai Hospital, University of Toronto, Toronto, ON M5S, Canada

${ }^{3}$ The Laboratory for Cognitive Neuroscience and Women's Health Department of Psychology, University of Toronto, Toronto, ON M5S, Canada

\begin{abstract}
Sex differences have been reported repeatedly in pain and response to opioid analgesia with women representing the majority of chronic non-cancer pain (CNCP) patients as well as a growing population of opioid users and misusers. However, none of these reports has investigated sex differences in the prevalence of overlapping multiple pain conditions (MPC), especially among patients under opioid therapy. Two hundred eighty-three charts were reviewed of patients attending a tertiary pain clinic with multiple sub-practices in a large Canadian city over a one-year period. 201 patients suffering from CNCP and under opioid therapy were selected. A significant sex difference was found in the number of patients, while no statistically significant sex difference was found in type of opioids prescribed. Moreover, significant sex differences were found in the prevalence and types of overlapping CNCP conditions as well as in the pattern of opioid misuse. With respect to overlapping pain conditions, Chronic pelvic pain (CPP) was the most common pain condition to co-occur in women, while in men, fibromyalgia was the most common. Taken together, all of these suggest that sex differences are significant in patients with overlapping CNCP conditions, and taking them into consideration might provide more comprehensive pain management.
\end{abstract}

Keywords: Chronic non-cancer pain (CNCP); CNCP conditions; Opioid treatments

\section{Introduction}

Chronic non-cancer pain (CNCP) is one of the most common reasons for seeking health care with one in five Canadians suffering from CNCP [1]. Moreover, CNCP accounts for up to $78 \%$ of visits to emergency care facilities, and usually leads to both a severe decline in the quality of life and a startling rise in the incidence of disability [2]. Successful treatments are few and far between with one of the most potent analgesics, opioid medications, associated consistently with concerns about problematic opioid use (POU) (i.e. misuse, abuse or addiction) [3-6].

Sex differences in both the incidence and types of chronic pain conditions have gained increasing attention as an area of research with the potential to lead to a better understanding the differences in CNCP patients' response to treatments [7-9]. Clinical pain studies have reported repeatedly that women represent the majority of patients suffering from CNCP conditions like Fibromyalgia, Chronic Headaches; Temporomandibular disorder (TMD) Pain; and Chronic Pelvic Pain (CPP) $[2,7,10]$. All of the CNCP conditions are often treated with opioids and, as with pain, the available human studies have reported a significantly greater analgesic effect in women in comparison to men [11-15]. Moreover, recent studies report that women now represent a growing population suffering from POU after long-term opioid treatment $[12,16-18]$

Based on these findings, sex differences would seem especially pertinent in understanding both the ra es and treatments for CNCP. However, work in this area consists of studies investigating of only one CNCP condition at a time [19]. This approach leaves a serious gap in our understanding because it is well known that $\mathrm{CNCP}$ conditions most often co-occur as a constellation of multiple pain conditions (MPC) with overlapping symptoms [19-25]. Moreover, most studies investigating sex differences in clinical pain have not controlled for the current treatments participants might be prescribed especially with respect to opioids or an often note treatment outcome, POU [19]. Consequently, we decided to review charts of CNCP patients under opioid medication in order to determine whether there are sex differences in the prevalence of overlapping MPC and if so, the constellation of types of condition between women and men under opioid therapy.

\section{Methods}

After obtaining approval from the Research Ethics Boards of both the University of Toronto and Mount Sinai Hospital, a chart review was conducted of all patients under opioid treatment referred to three different practices at the Wasser Pain Management Centre, a tertiary pain clinic in Toronto, ON from July 1, 2012 until July 31, 2013. Two hundred eighty-three charts were reviewed in total; 82 patients were excluded because they were ineligible (10 patients had cancer and 72 CNCP patients were not using opioids 56 (87\%) of which were women), leaving 201 patients with CNCP under opioid treatment. If patients could be cross-referred to more than one practice, they were counted only once.

Data collected included: sex, age, pain condition or conditions, type of treatment, duration of pain condition, responses to the long form McGill Pain Questionnaire (Pain Rating Index, PRI) [26], Pain Intensity Scale scores [27], Pain Catastrophizing Scale scores [28], history of surgery or trauma, types of pain medication, Hamilton Anxiety Scale (HAS) [29], Beck Depression Inventory (BDI) [30], history of drug abuse, and whether or not a patient was diagnosed with POU.

*Corresponding author: Samah Hassan, MD, MSc, Institute of Medical Science University of Toronto, Canada, Tel: +16474698447; E-mail: sam.hassan@mail. utoronto.ca

Received December 22, 2015; Accepted January 18, 2016; Published January 23, 2016

Citation: Hassan S, Gordon A, Einstein G (2016) Sex Differences in Overlapping Chronic Non- cancer Pain Conditions in a Tertiary Pain Clinic. Pain Manage Med 2: 110 .

Copyright: $\odot 2016$ Hassan S, et al. This is an open-access article distributed under the terms of the Creative Commons Attribution License, which permits unrestricted use, distribution, and reproduction in any medium, provided the original author and source are credited. 


\section{Statistical analysis}

Data were analyzed using SPSS (SPSS 15.0.1, 2006, Chicago Illinois. Software Inc.). Means and standard deviations were calculated for the above patient characteristics in the form of continuous numerical variables. In order to study sex differences between nominal variables, a comparison between female and male patients with CNCP was made using a Student $\mathrm{t}$-test and Pearson Chi-square test for non-parametric data. P-values of $<0.05$ were considered significant.

\section{Results}

\section{Patient characteristics}

A total of 283 charts comprising a mix of women and men were reviewed. Women were disproportionately represented in this pain clinic, 185 (68\%) patients were women, while 88 (32\%) were men.

Each chart was examined for the types of treatments patients were given. Therapies varied both in type and in modalities. On average, patients had been prescribed six different kinds of treatment, ranging from antidepressant medications (Cymbalta; duloxetine, serotoninnorepinephrine reuptake inhibitor (SNRI), Elavil; amitriptyline, tricyclic antidepressants), and anticonvulsants (Lyrica; pregabalin, gabapentin, carbamazepine; anticonvulsant and mood stabilizer) to nerve blocks, local steroid injections, Botox injections, physiotherapy, massage, acupuncture, psychotherapy, mindfulness, chiropractor, and aggressive treatment such as opioids.

During this year, 201 patients were under opioid treatment and 88 were not. Oxycodone was the most prescribed opioid for both female and male patients, followed by morphine, methadone and fentanyl. While, no statistically significant difference was found between men and women in type of opioids prescribed, a significant sex difference was found in the number of CNCP patients under opioid treatment $(n=201)$, (women: $n=129$ (64\%); men: $n=72$ (36\%) $\left(\chi^{2}=32.677 d f=1\right.$, $\mathrm{p}<0.01)$. Moreover, out of the total of 201 patients under opioid treatment, of the 79 suffering from POU, 56\% were women and $44 \%$ were men. Interestingly, although no significant sex difference was found in the percentage of patients with $\operatorname{POU}\left(\mathrm{x}^{2}=4.074, \mathrm{df}=1, \mathrm{p}=0.44\right)$, men were significantly more likely to have a history of drug abuse compared to women: $44 \%$ of men and only $19 \%$ of women $\left(\chi^{2}=14.035\right.$, $\mathrm{df}=1, \mathrm{p}<0.01)$ (Figure 1).

Women were significantly more likely than men to have a history of surgery (women, $n=42$, men $n=21 \chi^{2}=37.977, d f=1, p<0.01$ ). In fact, $66 \%$ of those women had surgery in the abdominopelvic area. However, no sex differences were found on the PRI, Pain Intensity Scale scores, or Pain Catastrophizing Scale scores. Similarly, no sex differences were found either on the BDI or the HAS.

In spite of the sex differences found in some of the characteristics, there was no difference in the type of information gathered from women or men in the charts in that the charts contained no information about number of births, menstrual cycle irregularities, or any menstrual cycle link with pain severity (Table 1).

\section{Prevalence and Types of MPC}

A total of 119 (59\%) patients presented with MPC with a statistically significant sex difference: of 129 women, $100(78 \%)$ reported MPC, while of 72 men, only $19(26 \%)$ did so $\left(\chi^{2}=50.017, \mathrm{df}=1, \mathrm{p}<=0.01000\right)$ (Figure 1).

There were, also, sex differences in the constellation of pain conditions comprising the MPC. In women, CPP was the most

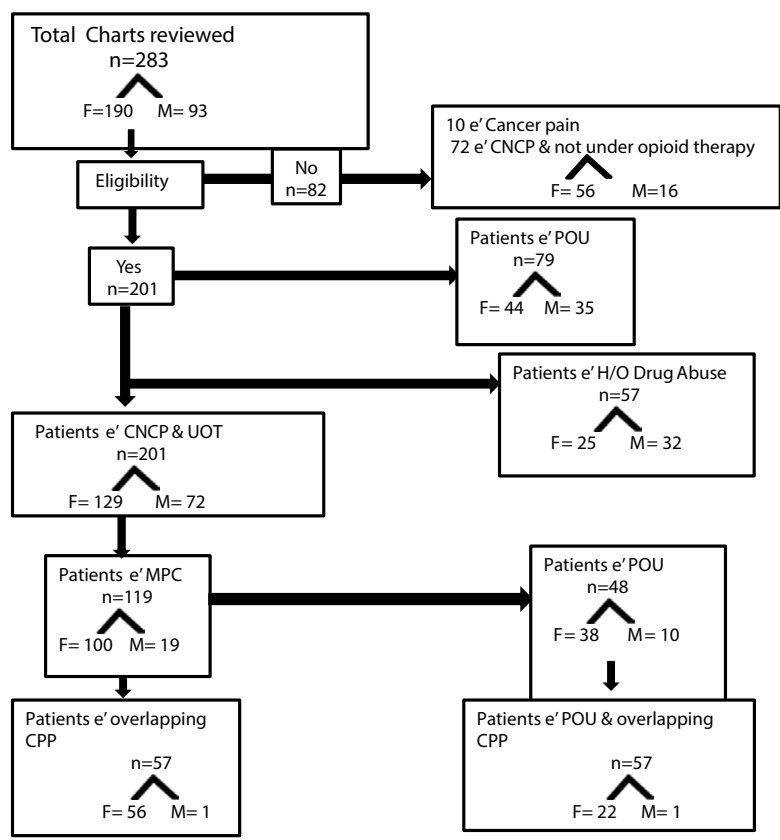

Figure 1: Chart Review Flow. (F: Female, M: Male, E': With, CNCP: Chronic Non Cancer Pain, POU: Problematic Opioid Use, H/O: History Of, UOT: Under Opioid Therapy, MPC: Multiple Pain Conditions, CPP: Chronic Pelvic Pain).

common co-occurring condition (56\%) followed by fibromyalgia (49\%), LBP (33\%), chronic headaches (29\%) and TMD (16\%). In contrast, in men, fibromyalgia was the most common co-occurring CNCP (68\%) followed by chronic headaches (53\%), TMD (47\%), LBP (42\%), and CPP (5\%). The sex difference in the most common overlapping condition was significant $\left(\chi^{2}=16.470, \mathrm{df}=1, \mathrm{p}<0.01\right.$ ) (Table 2). Notably, in women, endometriosis and/or irritable bowel syndrome (IBS) together were the most common reasons for co-occurring CPP in women with 36 out of 56 (64\%) having one or both of these conditions.

\section{Note that the full percentages do not all add up to $100 \%$ as the CNCP conditions are overlapping}

Moreover, $38 \%$ of the women suffering from MPC had POU while $53 \%$ of the men had POU. In women with POU, CPP was also the most common co-occurring condition (58\%), while fibromyalgia and chronic headaches were equally the most represented co-occurring conditions in men with POU (50\%) (Figure 1).

\section{Relationship of chronic pain to reproductive years}

The mean age of female patients at their initial visit was $42(\mathrm{SD} \pm 13$ ) and male patients was $45(\mathrm{SD} \pm 15)$. While there was no sex difference in the mean age between patients, when the range of ages were divided into groups: those who were 18-48 (as a proxy for women's reproductive years) and 49-71 (as a proxy for women in menopause), we found that women in their reproductive years (18-49) represented 90\% ( $\mathrm{n}=95)$ of the women with MPC, $75 \%(n=33)$ of women with POU and $79 \%$ $(n=53)$ of women with co-occurring CPP. For men who had MPC, $\mathrm{POU}$, or co-occurring CPP, there was no difference between the two age groups.

\section{Discussion}

This retrospective chart review is the first chart review of CNCP patients that includes a comparison by sex in the prevalence of 


\begin{tabular}{|c|c|c|}
\hline & Women & Men \\
\hline Pain Intensity: Mean(SD) & $7( \pm 2)$ & $6( \pm 2)$ \\
\hline $\begin{array}{c}\text { McGill Pain Questionnaire (PRI): } \\
\text { Mean(SD) }\end{array}$ & $33( \pm 13)$ & $32( \pm 12)$ \\
\hline $\begin{array}{c}\text { Pain Catastrophizing Scale: } \\
\text { Mean(SD) }\end{array}$ & $25( \pm 14)$ & $25( \pm 14)$ \\
\hline Depression (BDI):Mean(SD) & $23( \pm 14)$ & $23( \pm 13)$ \\
\hline Anxiety (HAS): Mean(SD) & $8( \pm 6)$ & $9( \pm 7)$ \\
\hline *H/O of surgery: N (\%) & $108(84 \%)$ & $30(42 \%)$ \\
\hline
\end{tabular}

*Statistically significant sex difference, SD: Standard Deviation. Table 1: Patient characteristics.

\begin{tabular}{|c|c|c|}
\hline & $\begin{array}{c}\text { Women } \\
\mathbf{n = 1 2 9}\end{array}$ & $\begin{array}{c}\text { Men } \\
\mathbf{n = 7 2}\end{array}$ \\
\cline { 2 - 3 } & $\begin{array}{c}\text { MPC } \\
\mathbf{n = 1 0 0} \\
\mathbf{n}(\mathbf{\%})\end{array}$ & $\begin{array}{c}\text { MPC } \\
\mathbf{n = 1 9} \\
\mathbf{n}(\%)\end{array}$ \\
\hline & $56(56)$ & $1(5)$ \\
\hline CPP & $16(16)$ & $9(47)$ \\
\hline TMD & $49(49)$ & $13(68)$ \\
\hline Fibromyalgia & $33(33)$ & $8(42)$ \\
\hline LBP & $29(29)$ & $10(53)$ \\
\hline
\end{tabular}

MPC: Multiple Pain Conditions, CPP: Chronic Pelvic Pain, TMD: Temporomandibular Joint Disorder, LBP: Low Back Pain

Table 2: Frequencies of individual pain conditions in patients with MPC.

overlapping of CNCP conditions in women and men under opioid therapy. We consider this an important first step to understanding the different characteristics of patients presenting with CNCP to Tertiary Pain Clinics.

A total of 283 charts were reviewed across one year. Each chart was examined for the chronic pain conditions represented and the types of treatments patients were given. From the 283 charts, 201 patients were under opioid treatment. A significant sex difference was found in the number of patients under opioid treatment with women comprising $64 \%$ of those treated with opioids. No statistically significant difference was found between men and women in type of opioids prescribed for men and women with the most commonly prescribed being Oxycodone. It might, be useful to consider this sex difference in the future. It is possible that men on opioids might have had a better response to a different type since all the varieties prescribed in this clinic are known to act primarily at the $(\kappa)$ receptor and while women tend to respond better to $\kappa$ opioid drugs, men tend to respond better to $\mathrm{mu}(\mu)$ opioids [31,32]. While there was no significant sex difference in the percentage of patients with POU, in men, the transition to POU might be explained by the fact that $44 \%$ of them already had a history of drug abuse and were thus more vulnerable to POU. As for women, they might have transitioned to POU despite the fact that they had no history of drug abuse, because opioid efficacy is altered by changes in the levels of ovarian hormones and thus at certain stages of the menstrual cycle, they might require higher doses for optimal relief.

Consistent with other studies, women were disproportionately represented in this clinic in general, and disproportionately represented in the opioid treatment group. Nevertheless, no sex differences were found in pain, depression, anxiety and catastrophizing scales. This is interesting since women are believed to have higher pain, depression, anxiety and catastrophizing scores in general [9]. One explanation for this lack of sex difference might be that the chronicity of the pain conditions, especially in women, masks sex differences in the scales. Another is perhaps because both men and women were under psychotropic treatments which again, may abolish sex differences. It may also be the case that once a person has a chronic pain condition the condition, itself, equalizes the likelihood of depression. This is an area that would benefit from further study.

This study also corroborated overwhelming that CNCP conditions rarely occur as single condition but rather as MPC with overlapping symptoms spanning different pain conditions. In the clinic we studied, these overlapping conditions included chronic headaches, TMD, fibromyalgia and CPP. Interestingly, overall, CNCP patients were disproportionately women who were also disproportionally affected by MPC when compared to men.

With respect to the constellation of MPC, at least in this clinic, CPP was the most commonly co-occurring pain syndrome in women. Interestingly, when we studied the type of co-occurring CPP, 64\% of those women had CPP due to endometriosis and/ or IBS, which have been repeatedly reported in many studies as estrogen-sensitive conditions [33-36]. This chart review also revealed that the most of the women with MPC and CPP were in their reproductive years and approximately $66 \%$ of the women with MPC and CPP had a history of abdomino-pelvic surgery, including hysterectomy, oophorectomy and laparoscopy.

It is currently believed that peripheral sensitization due to endometriosis significantly influences pain processing via the connection between the central nervous system and the sensitized inputs from ectopic endometrial growths $[37,38]$. These endometrial growths can actually influence neuronal activity by modulating both the inhibitory and excitatory mechanisms that in turn modulate pain signals transferred to central pain areas [37,39]. Hence, while the charts could not report directly on the relation of the women's CNCP condition and their menstrual and reproductive status, nor their levels of ovarian hormones, the fact that CPP co-occurs with other CNCP conditions such as fibromyalgia, chronic migraines, TMD and LBP, suggests that estrogen might as well modulate other co-occurring conditions. The effects of hormonal variation might be important to follow up in these types of patients.

Additionally, the literature confirms that hormonal treatment specifically is of benefit for CPP associated with endometriosis $[19,25]$, thus suggesting the necessity of evaluation for the presence of cooccurring CPP condition especially among women with MPC and CPP within their reproductive years and the potential benefit of hormonal therapy for managing their pain [37,39-43]. Moreover, it is also known that opioid efficacy waxes and wanes with the cyclic variations in reproductive hormones, and since those women were on opioid treatment, it would be useful to better understand how the ovarian cycle, pain, and opioid efficacy interact to modulate CNCP in order to achieve more successful treatment in those women.

\section{Strengths and Weaknesses of the Study}

An important strength of this study is that it is the first chart review of a Tertiary Pain Clinic to look at the intersection of sex differences, multiple chronic pain conditions, and opioid treatment. Our findings suggest that more such studies should be done in order to obtain more generalizable knowledge and to verify the treatment recommendations below.

A weakness of this study is that there were so few men, as compared with women, being treated by this pain clinic in the year studied. This may be due to the particular focus of this pain clinic as well as to the already identified disproportionate burden of chronic pain for women. Never the less, with even a small sample of men it is possible to gain a 
snapshot of the types of their pain, the fact that they, too, present with overlapping pain conditions, and that they bear the brunt of a history of problematic opioid use while women bear the brunt of surgery. Another weakness of this study, as is the case for all chart reviews, is that it utilized data that were not originally collected for research purposes. Therefore, data were limited and were not collected prospectively in a standardized fashion. In spite of these limitations, however, this chart review provides a snapshot of how CNCP patients present at a clinical practice and opens further paths for future research capable of bringing new knowledge into practice.

\section{Recommendations}

A number of concrete recommendations for improved pain treatment for women and men emerge from our study. First, while abundant evidence demonstrates sex differences in pain, and opioid treatment, clearly more convergent evidence from appropriate longterm clinical trials is needed to provide the evidence necessary to adequately convince clinicians of whether or not and how CNCP is modulated by sex. Stronger evidence would clear the way for determining how treatment efficacy might depend on sex.

Second, we, also, recommend querying all patients upon intake regarding any association of overlapping pain conditions. The practice of triaging patients to practices dealing with only one pain condition before noting all the overlapping conditions in their charts limits the understanding of the whole pain condition. Whether CPP is one of the overlapping conditions should be queried directly of both women and men during the first steps of pain management and throughout follow up visits.

Additionally, adding some demographic questions such about ethnicity, work, and history of sexual abuse as well as other adverse childhood experiences, especially for those who have CPP would be helpful in understanding the larger context of the pain condition and might present treatment options $[44,45]$. As well, if CPP is present in women, it is important to determine whether its underlying cause is endometriosis.

\section{Conclusions}

These findings from one year's intake to a tertiary pain clinic demonstrate that women and men present differently with CNCP have different types of overlapping pain conditions, and develop POU differently. Our findings suggest that additional types of information should be gathered for women and men allowing the physician to better understand any correspondence between CNCP conditions and the menstrual cycle or hormone variation as well as any changes in treatment efficacy with the menstrual cycle. The type of opioid administered might also require specificity by sex. Taken together, considering the sex of a patient with the possible concomitant sex differences in the types of conditions, prevalence of multiple pain conditions, type of opioid administered, and an already established POU as well as the effect of varying hormone levels on opioid efficacy might improve the efficacy of treatment for both men and women with CNCP conditions.

\section{References}

1. Moulin DE, Clark AJ, Speechley M, Morley-Forster PK (2002) Chronic pain in Canada--prevalence, treatment, impact and the role of opioid analgesia. Pain Res Manag 7: 179-184

2. Todd $\mathrm{KH}$, Ducharme J, Choiniere M, Crandall CS, Fosnocht DE, et al. (2007)"Pain In the emergency department: results of the Pain And Emergency Medicine Initiative (PEMI) Multicentre Study." J Pain 8: 460-466.
3. Ashburn MA, Staats PS (1999) Management of chronic pain. Lancet 353: 18651869.

4. Ballantyne JC, Mao J (2003) Opioid therapy for chronic pain. N Engl J Med 349: 1943-1953.

5. Eriksen J, Sjøgren P, Bruera E, Ekholm O, Rasmussen NK (2006) Critical issues on opioids in chronic non-cancer pain: an epidemiological study. Pain 125: $172-179$.

6. McWilliams LA, Asmundson GJ (2007) The relationship of adult attachment dimensions to pain-related fear, hypervigilance, and catastrophizing. Pain 127 27-34.

7. Berkley KJ (1997) Sex differences in pain. Behav Brain Sci 20: 371-380.

8. Fillingim RB, King CD, Ribeiro-Dasilva MC, Rahim-Williams B, Riley JL 3rd (2009) Sex, gender, and pain: a review of recent clinical and experimental findings. J Pain 10: 447-485.

9. Fillingim RB, Ness TJ (2000) Sex-related hormonal influences on pain and analgesic responses. Neurosci Biobehav Rev 24: 485-501.

10. Greenspan JD, Craft RM, LeResche L, Arendt-Nielsen L, Berkley KJ, et al. (2007) Studying sex and gender differences in pain and analgesia: a consensus report. Pain 132 Suppl 1: S26-45.

11. Chen EH, Shofer FS, Dean AJ, Hollander JE, Baxt WG, et al. (2008) Gende disparity in analgesic treatment of emergency department patients with acute abdominal pain Acad Emerg Med 15: 414-418.

12. Cicero TJ, Nock B, O'Connor L, Meyer ER (2002) Role of steroids in sex differences in morphine-induced analgesia: activational and organizational effects. J Pharmacol Exp Ther 300: 695-701.

13. Craft RM, Mogil JS, Aloisi AM (2004) Sex differences in pain and analgesia: the role of gonadal hormones. Eur J Pain 8: 397-411.

14. Craft RM (2007) Modulation of pain by estrogens. Pain 132 Suppl 1: S3-12.

15. Craft RM (2003) Sex differences in drug- and non-drug-induced analgesia. Life Sci 72: $2675-2688$

16. Kelly MJ, Rønnekleiv OK, Ibrahim N, Lagrange AH, Wagner EJ (2002) Estrogen modulation of $\mathrm{K}(+)$ channel activity in hypothalamic neurons involved in the control of the reproductive axis. Steroids 67: 447-456.

17. Martin VT (2009) Ovarian hormones and pain response: a review of clinical and basic science studies. Gend Med 6 Suppl 2: 168-192.

18. Back SE, Lawson KM, Singleton LM, Brady KT (2011) Characteristics and correlates of men and women with prescription opioid dependence. Addict Behav 36: 829-834.

19. Hassan S, Muere A, Einstein G (2014) Ovarian hormones and chronic pain: A comprehensive review. Pain 155: 2448-2460.

20. Dao TT, Reynolds WJ, Tenenbaum HC (1997) Comorbidity between myofascial pain of the masticatory muscles and fibromyalgia. J Orofac Pain 11: 232-241.

21. McNeill C (1997) History and evolution of TMD concepts. Oral Surg Oral Med Oral Pathol Oral Radiol Endod 83: 51-60.

22. Itza F, Zarza D, Serra L, Gómez-Sancha F, Salinas J, et al. (2010) [Myofascial pain syndrome in the pelvic floor: a common urological condition]. Actas Uro Esp 34: 318-326.

23. John MT, Miglioretti DL, LeResche L, Von Korff M, Critchlow CW (2003) Widespread pain as a risk factor for dysfunctional temporomandibular disorder pain. Pain 102: 257-263.

24. Paras ML, Murad MH, Chen LP, Goranson EN, Sattler AL, et al. (2009) Sexua abuse and lifetime diagnosis of somatic disorders: a systematic review and meta-analysis. Jama 302: 550-561.

25. Vercellini P, Somigliana E, Vigano P, Abbiati A, Barbara G, et al. (2009) Chronic pelvic pain in women: etiology, pathogenesis and diagnostic approach. Gynecol Endocrinol 25: 149-158.

26. Melzack R (1987) The short-form McGill Pain Questionnaire. Pain 30: 191-197.

27. Hawker GA, Mian S, Kendzerska T, French M (2011) Measures of adult pain: Visual Analog Scale for Pain (VAS Pain), Numeric Rating Scale for Pain (NRS Pain), McGill Pain Questionnaire (MPQ), Short-Form McGill Pain Questionnaire (SF-MPQ), Chronic Pain Grade Scale (CPGS), Short Form-36 Bodily Pain Scale (SF-36 BPS), and Measure of Intermittent and Constant Osteoarthritis Pain (ICOAP). Arthritis care \& research 63: S240-252. 
28. Stratton P, Berkley KJ (2011) Chronic pelvic pain and endometriosis: translational evidence of the relationship and implications. Hum Reprod Update 17: $327-346$

29. Hamilton M (1969) Diagnosis and rating of anxiety. Br J Psychiatry 3: 76-79.

30. Beck AT, Steer RA, Brown GK, Ghisi M (2006) BDI-2: Beck depression inventory-2.: manuale: OS, Organizzazioni speciali.

31. Gear RW, Gordon NC, Heller PH, Paul S, Miaskowski C, et al. (1996) Gender difference in analgesic response to the kappa-opioid pentazocine. Neurosci Lett 205: 207-209.

32. Gear RW, Miaskowski C, Gordon NC, Paul SM, Heller PH, et al. (1996) Kappaopioids produce significantly greater analgesia in women than in men. Nat Med 2: $1248-1250$.

33. Bernstein MT, Graff LA, Targownik LE, Downing K, Shafer LA, et al. (2012) Gastrointestinal symptoms before and during menses in women with IBD. Aliment Pharmacol Ther 36: 135-144.

34. Chang L, Lee OY, Naliboff B, Schmulson M, Mayer EA (2001) Sensation of bloating and visible abdominal distension in patients with irritable bowe syndrome. The American journal of gastroenterology 96: 3341-3347.

35. Heitkemper MM, Cain KC, Jarrett ME, Burr RL, Hertig V, et al. (2003) Symptoms across the menstrual cycle in women with irritable bowel syndrome. Am J Gastroenterol 98: 420-430.

36. Matheis A, Martens U, Kruse J, Enck P (2007) Irritable bowel syndrome and chronic pelvic pain: a singular or two different clinical syndrome? World $\mathrm{J}$ Gastroenterol 13: 3446-3455.

37. Simoni-Wastila L1 (2000) The use of abusable prescription drugs: the role of gender. J Womens Health Gend Based Med 9: 289-297.

38. Kitawaki J, Kado N, Ishihara H, Koshiba H, Kitaoka Y, et al. (2002) Endometriosis: the pathophysiology as an estrogen-dependent disease. The Journal of steroid biochemistry and molecular biology 83: 149-155.

39. Howard FM (2003) The role of laparoscopy in the chronic pelvic pain patient Clin Obstet Gynecol 46: 749-766.

40. Huang HY (2008) Medical treatment of endometriosis. Chang Gung Med J 31 : 431-440.

41. Miaskowski C, Gear RW, Levine JD (2000) Sex-related differences in analgesic responses.

42. Turk DC, Wilson HD, Cahana A (2011) Treatment of chronic non-cancer pain Lancet 377: 2226-2235.

43. Unruh AM (1996) Gender variations in clinical pain experience. Pain 65: 123 167.

44. Mailis-Gagnon A, Yegneswaran B, Nicholson K, Lakha SF, Papagapiou M, et al (2007) Ethnocultural and sex characteristics of patients attending a tertiary care pain clinic in Toronto, Ontario. Pain Res Manag 12: 100-106.

45. Paller CJ, Campbell CM, Edwards RR, Dobs AS (2009) Sex-based differences in pain perception and treatment. Pain Med 10: 289-299. 